\title{
Comparison of the properties of neural stem cells of the hippocampus in the tree shrew and rat in vitro
}

\author{
YUAN-DONG HU ${ }^{1,2^{*}}$, QIONG ZHAO ${ }^{3 *}$, XUE-RONG ZHANG ${ }^{3}$, LIU-LIN XIONG $^{1}$ \\ ZI-BIN ZHANG ${ }^{1}$, PIAO ZHANG ${ }^{2}$, RONG-PING ZHANG $^{2 *}$ and TING-HUA WANG ${ }^{1,2 *}$ \\ ${ }^{1}$ Institute of Neurological Disease, West China Hospital, Sichuan University, Chengdu, Sichuan 610041; \\ ${ }^{2}$ Institute of Neuroscience, Kunming Medical University, Kunming, Yunnan 650031; ${ }^{3}$ Department of Anesthesiology, \\ Sun Yat-Sen Memorial Hospital, Sun Yat-Sen University, Guangzhou, Guangdong 510120, P.R. China
}

Received April 20, 2017; Accepted December 1, 2017

DOI: $10.3892 / \mathrm{mmr} .2018 .8589$

\begin{abstract}
Neural stem cells (NSCs) are characterized by the ability of self-renewal and capacity to proliferate and produce new nervous tissue. NSCs are capable of differentiating to three lineages of neural cells, including neurons, oligodendrocytes and astrocytes. Furthermore, hippocampal NSCs transplantation can improve the neurological deficits associated with expression of cytokines. Therefore, to compare the properties of NSCs of tree shrews and rats in vitro, NSCs from tree shrews (tsNSCs) and rats $\mathrm{f}(\mathrm{rNSCs})$ were isolated. Nestin was used as a marker to identify the cultured NSCs. Neuronal nuclei protein and glial fibrillary acidic protein (GFAP) were utilized to demonstrate the differentiation of NSCs towards neurons and astrocytes, respectively, in vitro. Furthermore, the expression of neurotrophin 3 (NT3), brain-derived neurotrophic factor (BDNF), glial cell-derived neurotrophic factor (GDNF) and transforming growth factor (TGF) $\beta 1$ was also investigated in tsNSCs and rNSCs. The expression of all of the aforementioned proteins was detected using immunofluorescence methods. The results demonstrated that, after 5 days of culture, the average number of neurospheres in the cultured tsNSCs was significantly lower compared with rNSCs $(\mathrm{P}=0.0031)$. Additionally, compared with the rNSCs, tsNSCs exhibited an enhanced differentiation ability towards neurons. Furthermore, the expression of NT3 in the tsNSCs was higher compared with rNSCs $(\mathrm{P}<0.01)$, while the expression of BDNF
\end{abstract}

Correspondence to: Professor Rong-Ping Zhang, Institute of Neuroscience, Kunming Medical University, 1168 Chun Rong Road, Kunming, Yunnan 650031, P.R. China

E-mail: zhrpkm@163.com

Professor Ting-Hua Wang, Institute of Neurological Disease, West China Hospital, Sichuan University, 17 Section 3 of Renmin South Road, Chengdu, Sichuan 610041, P.R. China

E-mail: tinghua_neuron@263.net

${ }^{*}$ Contributed equally

Key words: tree shrew, rat, neural stem cells, immunohistochemistry was lower $(\mathrm{P}=0.045)$. However, no significant differences were observed in the expression level of GDNF and TGF $\beta 1$ between rNSCs and tsNSCs. Therefore, these results indicate that tsNSCs exhibit specific characteristics that are different from rNSCs, which provides novel information for the understanding of NSCs obtained from tree shrews. Overall, the results of the current study provide evidence to support the increased application of tree shrews as models for diseases of the central nervous system.

\section{Introduction}

Neural stem cells (NSCs) are a type of stem cell that possess self-renewal, self-replication and multi-differentiation properties. Under certain conditions, NSCs may be induced to differentiate into neurons, astrocytes and oligodendrocytes (1-3). It has been demonstrated that NSCs have important roles in the replacement, recovery, and neurotrophy and immunoregulation (4). NSCs promote the recovery of animals with motion, sensory and cognitive dysfunction to a certain extent (5-8). Therefore, NSCs may have wide applications in clinical practice.

Several studies have demonstrated that NSCs are resident in various areas of the rat brain, including the hippocampus, cerebral hemisphere, hindbrain, spinal cord, cerebral ventricle area in the lateral ventricles, subventricle area and the cerebral cortex $(9,10)$. For the source of cultured NSCs in vitro, previous reports have established methods for the separation and culture of NSCs derived from the hippocampus of embryos in rats, mice, crab-eating macaques and humans; the cultured NSCs were cultured successfully in vitro and the cultured NSCs were induced to differentiate into neurons and glial cells (11-14). However, regarding research on central nervous system (CNS) diseases, rats and mice are rodents, and there are substantial differences between rodent and primate models. Crab-eating macaques have certain disadvantages, including high cost, difficult to breed and the use of fewer animals is permitted. Furthermore, ethical issues are associated with the use of human embryos. Therefore, it is necessary to identify more suitable experimental animals as a source of NSCs in in vitro models.

Tree shrews exhibit various characteristics that are similar to humans, including their biological features, metabolism, 
physiology, biochemistry and genome. Therefore, tree shrews are considered as a type of novel experimental animal model that may partially replace the primate models $(15,16)$. Due to the developed brain of the tree shrew, it is primarily employed for studies concerning the nervous system and the preparation of models of nervous system diseases (17-19). The use of the tree shrew as an animal model has attracted increasing attention and researches have already obtained useful results (20-23). However, little information exists concerning the differences between the stem cell origins of tree shrew NSCs (tsNSCs) and rat NSCs (rNSCs) Determining whether tsNSCs have identical or different properties to rNSCs is crucial for the application of tsNSCs.

In the present study, the features of NSCs derived from rats and tree shrews were compared. Furthermore, the expression of certain growth factors was also compared, with the aim of increasing the understanding of the biological characteristics of tsNSCs and improving their application in research.

\section{Materials and methods}

Animals andethicalstatement.Pregnant(E16) Sprague-Dawley rats (4 months old, $n=3$ ) and pregnant (E38) tree shrews ( 8 months old, $n=3$ ) of clean grade, weighing $170 \mathrm{~g}$, were used in the present study. All animals were provided by the Animal Experimental Center of Kunming Medical University (Kunming, China). Animal care and all experimental protocols were approved by the guidelines of the Institutional Medical Experimental Animal Care Committee of Sichuan University, West China Hospital, (Chengdu, China). Guidelines for Laboratory Animal Care and Safety from the National Institutes of Health were also followed (24). The animals were bred in separate cages in a temperature $\left(20 \pm 5^{\circ} \mathrm{C}\right), \mathrm{CO}_{2}(0.03 \%)$ and humidity (40-60\%)-controlled room with a $12 \mathrm{~h}$ light/dark cycle and free access to pellet chow and water.

Sample harvesting. Pregnant (E16) Sprague-Dawley rats and pregnant tree shrews (E38) were sacrificed after being anesthetized by intraperitoneal injection of $3.6 \%$ chloral hydrate $(1 \mathrm{ml} / 100 \mathrm{~g})$. Following rinsing in $75 \%$ ethanol for 3 min, the embryonic rat and tree shrews were removed under sterile conditions and kept in a culture dish containing Hank's balanced salt solution (Thermo Fisher Scientific, Inc., Waltham, MA, USA) on ice. The skulls were dissected and the brain hemisphere was removed, subsequently, the brain tissues were placed into pre-cooled PBS. Under an anatomic microscope, the meninges, olfactory bulb, cerebellum and brain stem were attentively removed and the hippocampal tissues were exposed and harvested. The samples were washed twice with pre-cooled PBS and placed into centrifuge tubes (25).

Preparation of single cell suspension. Hippocampal tissues were sheared into $1 \mathrm{~mm}^{3}$ sized tissue blocks. Trypsin $(0.25 \%$; 1:250; EMD Millipore, Billerica, MA, USA) was used to digest the tissue block at room temperature for $20 \mathrm{~min}$. The digested tissue solution was collected and placed in a $15-\mathrm{ml}$ centrifuge tube and Dulbecco's modified Eagle's medium/F12 (DMEM/F12; 1:1; Gibco; Thermo Fisher Scientific, Inc.) containing $10 \%$ fetal calf serum (Thermo Fisher Scientific, Inc.) was added to stop the digestion. Centrifugation was subsequently performed at $560 \mathrm{x} \mathrm{g}\left(4^{\circ} \mathrm{C}\right)$ for $5 \mathrm{~min}$. The supernatant was discarded and the cell suspension was harvested using DMEM/F12 culture media (1:1; Gibco; Thermo Fisher Scientific, Inc.) supplemented with 2\% B-27 (Gibco; Thermo Fisher Scientific, Inc.), $20 \mathrm{ng} / \mathrm{ml}$ basic fibroblast growth factor (bFGF; R\&D Systems, Inc., Minneapolis, MN, USA), 20 ng/ml epidermal growth factor (EGF; R\&D Systems, Inc.), $2 \mathrm{mmol} / 1$ glutamine (Gibco; Thermo Fisher Scientific, Inc.), 10,000 U/1 penicillin and $10 \mathrm{mg} / \mathrm{l}$ streptomycin.

Cell inoculation. Cell density was determined in the cell suspension and the density was adjusted to $5 \times 10^{5} / \mathrm{ml}$. The cells were inoculated onto the culture plates or bottles and kept in an incubator containing $5 \% \mathrm{CO}_{2}$ at $37^{\circ} \mathrm{C}$. Half of the culture medium was replaced every other day.

NSC passage. At 7 days post-culture, the diameter of neurospheres was commonly $\sim 100 \mu \mathrm{m}$ and subculturing was performed. In detail, NSCs were digested using $0.25 \%$ trypsin (1:250, Sigma-Aldrich; Merck KGaA, Darmstadt, Germany) at $37^{\circ} \mathrm{C}$ for $10 \mathrm{~min}$ and DMEM/F12 (1:1; Gibco; Thermo Fisher Scientific, Inc., Waltham, MA, USA) containing serum was used to stop the digestion. NSC suspension was collected into a $15 \mathrm{ml}$ centrifuge tube. Subsequently, centrifugation at $560 \mathrm{x} \mathrm{g}\left(4^{\circ} \mathrm{C}\right)$ was performed for $5 \mathrm{~min}$. The supernatant was discarded. The cell suspension was resuspended in DMEM/F12 (1:1; Gibco; Thermo Fisher Scientific, Inc.) containing 2\% B-27 (Gibco; Thermo Fisher Scientific, Inc.), 20 ng/ml bFGF (R\&D Systems, Inc.), $20 \mathrm{ng} / \mathrm{ml}$ EGF (R\&D Systems, Inc.), 2 mmol/1 glutamine (Gibco; Thermo Fisher Scientific, Inc.), 10,000 U/1 penicillin and $10 \mathrm{mg} / 1$ streptomycin. The cellular density was adjusted to $1.5-2.5 \times 10^{6} / \mathrm{ml}$ and inoculated into a culture bottle ( $25 \mathrm{ml}$ in volume), which was gently swayed for even distribution. The cells were incubated in an incubator at $37^{\circ} \mathrm{C}$.

Morphological observation. During the primary and secondary culture of cells derived from the hippocampus of rats and tree shrews, inverted phase contrast microscopy (Leica Microsystems GmbH, Wetzlar, Germany) was employed to observe and record the morphology and growth of NSCs. Prior to observation, cells were fixed with $4 \%$ paraformaldehyde for $20 \mathrm{~min}$ at room temperature.

Identification and differentiation of NSCs in vitro and the detection of neurotrophic factors by immunofluorescence. In order to confirm the cultured NSCs and detect the expression of neurotrophin 3 (NT3), brain-derived neurotrophic factor (BDNF), glial cell-derived neurotrophic factor (GDNF) and transforming growth factor (TGF) $\beta 1$, NSCs were cultured to the third generation, then immunofluorescence staining of nestin (a maker of NSCs) and neurotrophic factors (NT3, BDNF, GDNF and TGF $\beta 1$ ) was performed. For detecting the differentiation of NSCs, immunofluorescence staining of neuronal nuclei protein (NeuN; a neuronal marker) and glial fibrillary acidic protein (GFAP; an astrocyte marker) was performed to identify the characteristics of NSCs following serum induction (DMEM supplemented with 10\% fetal bovine serum (Gibco; Thermo Fisher Scientific, Inc.)) for $48 \mathrm{~h}$. The antibodies used were showed in Table I. Briefly, after discarding the culture medium of the third passage of NSCs, $0.25 \%$ trypsin 
Table I. Primary antibody details.

\begin{tabular}{llcc}
\hline Primary antibody & Company & Concentration & Catalog number \\
\hline BDNF & Boster & $1: 50$ & MGC34632 \\
NT-3 & Abcam & $1: 50$ & ab65804 \\
GDNF & ZhongShanJinQiao & $1: 100$ & EIA-1067 \\
TGF $\beta 1$ & Abcam & $1: 100$ & ab92486 \\
GFAP & ZhongShanJinQiao & $1: 50$ & ZA-0117 \\
NeuN & Abcam & $1: 100$ & ab177487 \\
Nestin & Abcam & $1: 50$ & ab92391 \\
\hline
\end{tabular}

All primary antibodies were rabbit antibodies. BDNF, brain-derived neurotrophic factor; NT3, neurotrophin 3; GDNF, glial cell-derived neurotrophic factor; TGF, transforming growth factor; GFAP, glial fibrillary acidic protein; NeuN, neuronal nuclei protein.

(1:250, Sigma-Aldrich; Merck KGaA) was added. Inverted phase contrast microscopy (Leica Microsystems GmbH, Wetzlar, Germany) was employed to observe the morphology of the NSCs. The majority of the cells were round in shape with cytoplasmic retraction and were loosened and floating. An appropriate amount of culture medium containing $10 \%$ fetal bovine serum was added to stop the digestion. The cell suspension was gently blended for even distribution using pap dropper. Subsequently, the cell suspension was transferred to 6-well plates and dropped onto sterile cover slips. The 6-well plates containing $1.2 \times 10^{6}$ per well NSCs were incubated in an incubator at $37^{\circ} \mathrm{C}$ for $4 \mathrm{~h}$. After 5 days of culture, tsNSCs and rNSCs were fixed with $4 \%$ paraformaldehyde for $30 \mathrm{~min}$ at room temperature, rinsed with 0.01 M PBS and incubated with 3\% goat serum (Gibco; Thermo Fisher Scientific, Inc.) for $30 \mathrm{~min}$ at $37^{\circ} \mathrm{C}$ to quench non-specific binding. Subsequently, cells were incubated overnight at $4^{\circ} \mathrm{C}$ with primary antibodies (Table I). As for the control group, the primary antibody was substituted with 0.01 M PBS. The glass slides were washed with $0.01 \mathrm{M}$ PBS three times, each for $2 \mathrm{~min}$, which was followed by incubation with cyanine 3-labeled anti-rabbit secondary antibody (1:200; cat. no. 111-165-003; Jackson Laboratory, Ben Harbor, ME, USA) at $37^{\circ} \mathrm{C}$ for $30 \mathrm{~min}$. Sections were observed under a fluorescent microscope. DAPI was used to counterstain the nuclei and the images were acquired using a Leica AF6000 cell station (Leica Microsystems GmbH). Finally, the proportion of positive cells was quantified using Image-Pro Plus 6.0 software (Media Cybernetics, Inc., Rockville, MD, USA). Each detection involved the preparation of six plates (6-well plates) of cells and each well was put into one sterile cover slip. Three random fields were selected per slide and evaluated by three investigators blinded to the experimental information, and the mean proportion of positive cells for each detection was calculated.

Counting the number of neurospheres. To investigate the expansion rates, tsNSCs and rNSCs were seeded in 6-well plates $\left(1.2 \times 10^{6}\right.$ per well) and cultured at $37^{\circ} \mathrm{C}$ for up to $72 \mathrm{~h}$. Neurospheres formed within 2-3 days in vitro. The aforementioned culture medium: DMEM/F12 supplemented with $2 \%$ B-27, $20 \mathrm{ng} / \mathrm{ml} \mathrm{bFGF}, 20 \mathrm{ng} / \mathrm{ml}$ EGF;, $2 \mathrm{mmol} / 1$ glutamin, $10,000 \mathrm{U} / 1$ penicillin and $10 \mathrm{mg} / 1$ streptomycin, was changed every 2 days. Numbers of neurospheres of tsNSCs and rNSCs were counted after culturing for $72 \mathrm{~h}$. The images were captured with a Leica AF6000 cell station (Leica Microsystems $\mathrm{GmbH})$. The number of neurospheres was quantified by using Image-Pro Plus 6.0 software (Media Cybernetics, Inc.). Five fields of vision were randomly selected per well and evaluated by three blinded investigators, and the mean number of neurospheres per well was calculated.

Statistical analysis. SPSS 18.0 software (SPSS, Inc., Chicago, IL, USA) was used for statistical analysis. Experimental data are presented as the mean + standard deviation and were analyzed by Student's t-test. $\mathrm{P}<0.05$ was considered to indicate a statistically significant difference.

\section{Results}

Growth of NSCs. Following the inoculation of tsNSCs and rNSCs, cells were well distributed under the microscope, cells commonly exhibited a round shape with a transparent cytoplasm. At 1 day after inoculation, the majority of cells were single celled and only a few exhibited a proliferative growth style. At this time, 2 or 4 cells connecting together was observed, and the cells were in a good growth state with good refraction and a transparent cytoplasm (Fig. 1). At day 3 of culture, the number of cell spheres increased and the size of cell spheres was uneven. Certain cells proliferated and formed embryonic spheres consisting of tens to several tens of cells. The cytoplasm of all the cell spheres was transparent. Occasionally, individual cells exhibited an adherent growth style with outgrowing processes (Fig. 1). At day 5 of culture, the volume of the cell spheres was enlarged in addition to the number of cell spheres. At this time, the majority of cells exhibited a suspended growth style, with regular morphology and strong refraction, without process outgrowth (Fig. 1).

Identification of NSCs. As revealed by immunofluorescence staining of nestin, primary cultured neurospheres from tree shrews and rats exhibited positive nestin staining. The cytoplasm of neurospheres exhibited a clear red color (Fig. 2), indicating strong positive nestin expression. DAPI staining stained the nuclei of NSCs blue (Fig. 2). Merged synthetic images demonstrated that nestin (red) and DAPI (blue) were co-localized in the cultured cells (Fig. 2). These results 

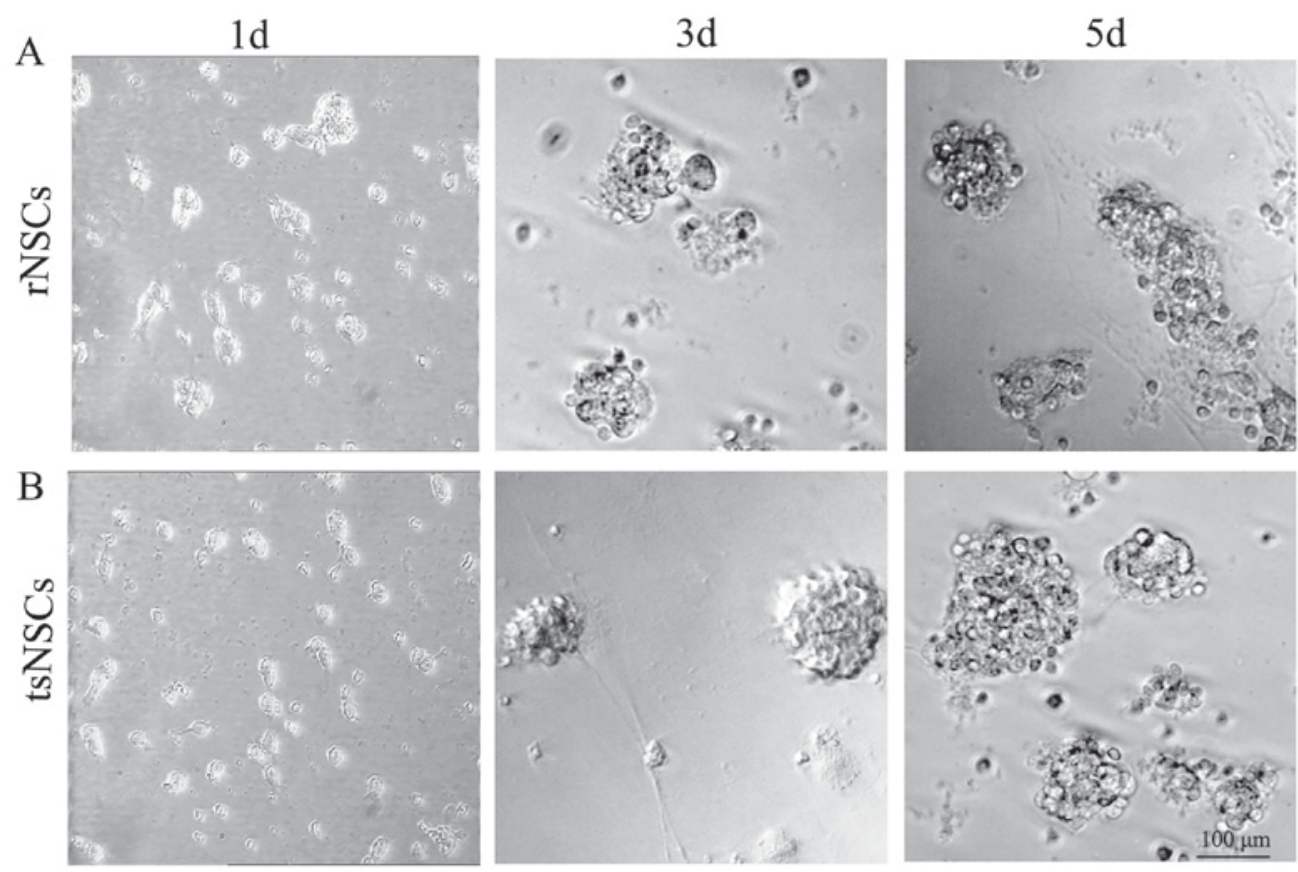

Figure 1. Morphology of NSCs in vitro following culture for 1, 3 and 5 days. (A) Primary cultures of (A) rNSCs and (B) tsNSCs at 1, 3 and 5 days of culture. Scale bar $=100 \mu \mathrm{m}$, applies to all images. NSCs, neural stem cells; rNSCs, rat NSCs; tsNSCs, tree shrew NSCs; d, days.
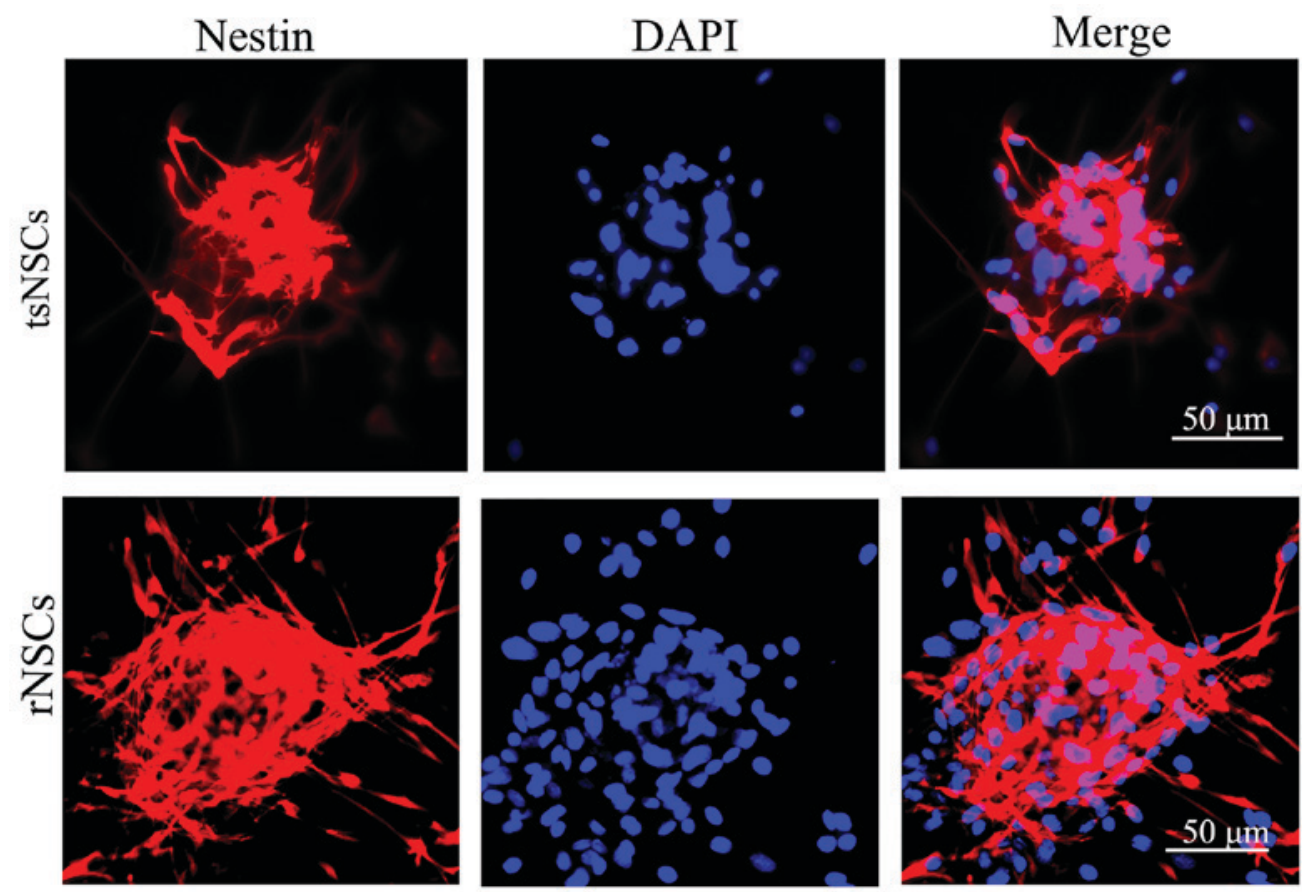

Figure 2. In vitro culture and identification of NSCs. Nestin staining (red fluorescence) was performed on tsNSCs and rNSCs on third passage cells. DAPI (blue fluorescence) was employed to stain nuclei. Scale bar $=50 \mu \mathrm{m}$, applies to all images. NSCs, neural stem cells; tsNSCs, tree shrew NSCs; rNSCs, rat NSCs.

indicate that the separation and culture tsNSCs and rNSCs was successful in the present study, which allowed subsequent experiments to be performed.

Measurement of NSC proliferation. At 5 days following inoculation in vitro, tsNSCs and rNSCs were present as cell colonies with round shapes (Fig. 3A). In order to detect the proliferation ability of rNSCs and tsNSCs, quantitative analysis of the average number of neurospheres demonstrated that the number of neurospheres in the cultured tsNSCs was significantly decreased compared with rNSCs ( $\mathrm{P}=0.0031$; Fig. 3B).

Detection of differentiation ability in NSCs cultured in vitro. At 5 days after induction by serum, certain NSCs exhibited radiate protuberance, and adherent and suspension culture was observed (Fig. 4A). Following immunofluorescence staining, 


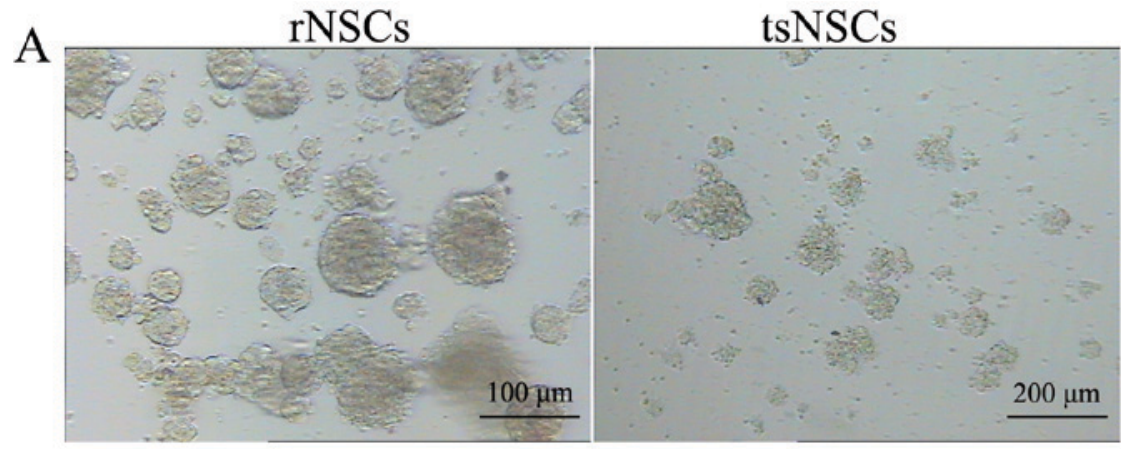

B

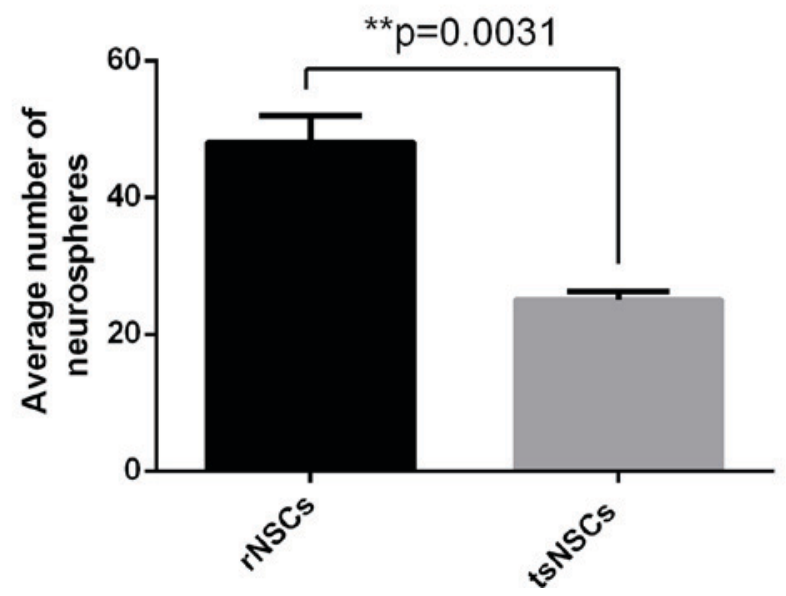

Figure 3. Comparison of neurosphere number in tsNSCs and rNSCs at 5 days following inoculation. (A) Representative images demonstrating the formation of neurospheres at 5 days following inoculation in the rNSC and tsNSC groups. (B) Quantitative analysis of the average number of neurospheres in the rNSC and tsNSC groups. Data are presented as the mean + standard deviation. ${ }^{* * *} \mathrm{P}<0.01$, as indicated. NSCs, neural stem cells; tsNSCs, tree shew NSCs; rNSCs, rat NSCs.

A

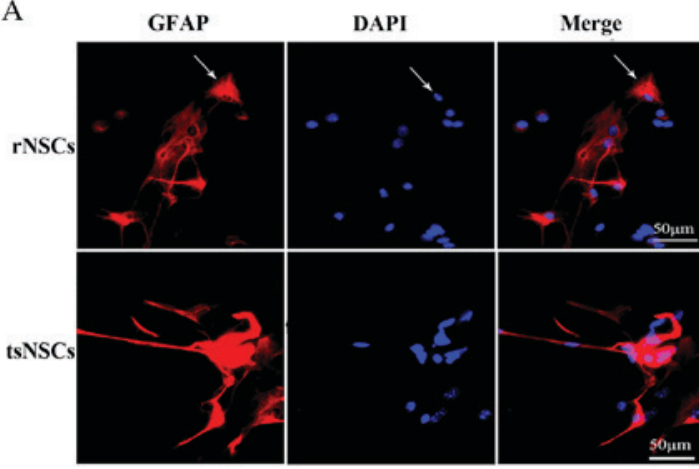

C

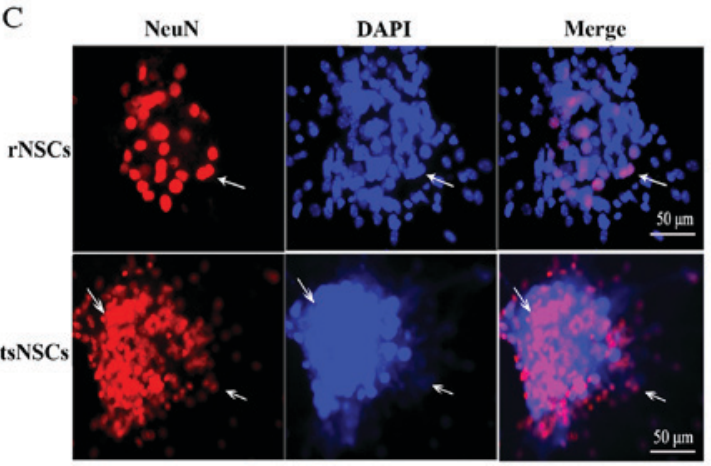

B

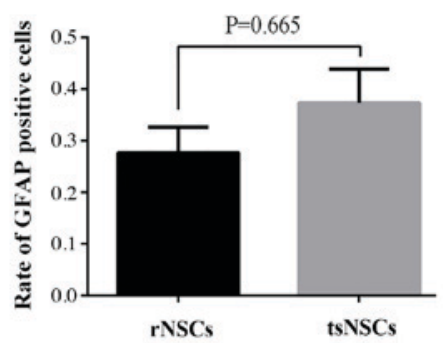

D

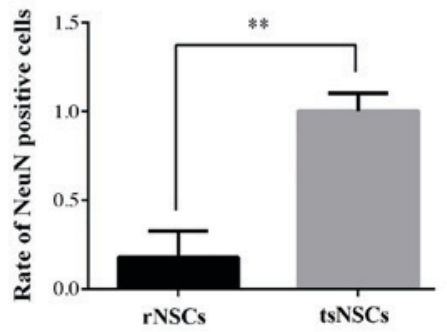

Figure 4. Comparison of the differentiation of tsNSCs and rNSCs into neurons and astrocytes. (A) Immunofluorescence staining of GFAP (red fluorescence) and nuclear staining with DAPI (blue fluorescence) in rNSCs and tsNSCs. Merged images are also presented. (B) Quantitative analysis of the proportion of GFAP positive cells. (C) Immunofluorescence staining of NeuN (red fluorescence) and nuclear staining with DAPI (blue fluorescence) in rNSCs and tsNSCs. Merged images are also presented. Examples of positive cells are indicated by white arrows. Scale bar, $50 \mu \mathrm{m}$. (D) Quantitative analysis of the proportion of GFAP positive cells. Data are presented as the mean + standard deviation. Scale bar $=50 \mu \mathrm{m}$, applies to all images. ${ }^{* *} \mathrm{P}<0.01$, as indicated. NSCs, neural stem cells; tsNSCs, tree shrew NSCs; rNSCs, rat NSCs; GFAP, glial fibrillary acidic protein; NeuN, neuronal nuclei protein. 

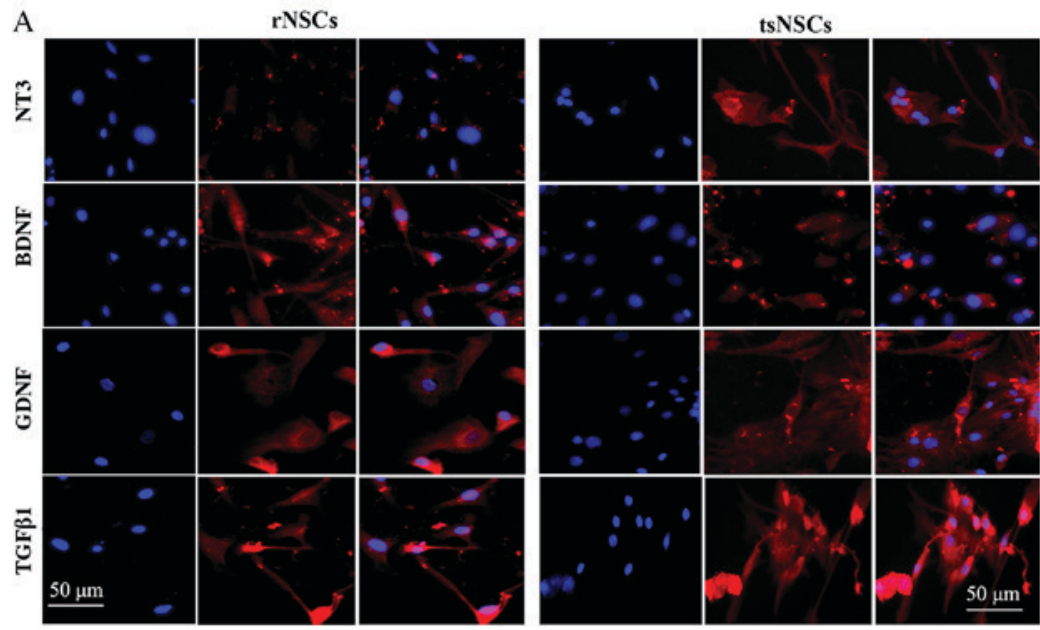

B

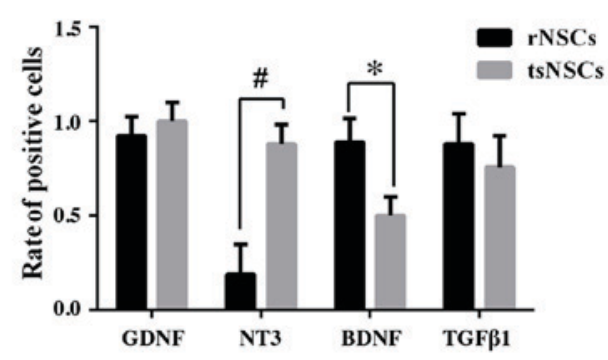

C

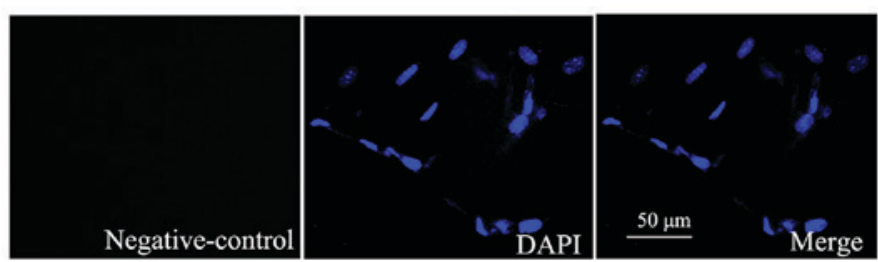

Figure 5. Expression of neurotrophic factors in tsNSCs and rNSCs. (A) Immunofluorescence staining of NT3, BDNF, GDNF and TGF $\beta 1$ in rNSCs and tsNSCs. Additionally, the left images are for DAPI staining, the middle images are for staining with NT3/BDNF/GDNF/TGF 31 and the right images are for merged DAPI and NT3/BDNF/GDNF/TGF $\beta 1$ staining. (B) Quantitative analysis of the proportion of NT3, BDNF, GDNF and TGF 31 positive rNSCs and tsNSCs. (C) Negative control for immunofluorescence staining, where PBS was employed instead of a primary antibody. Data are presented as the mean + standard deviation. Scale bar $=50 \mu \mathrm{m}$, applies to all images. ${ }^{*} \mathrm{P}<0.05$ and ${ }^{*} \mathrm{P}<0.01$, as indicated. NSCs, neural stem cells; tsNSCs, tree shrew NSCs; rNSCs, rat NSCs; NT3, neurotrophin 3; BDNF, brain-derived neurotrophic factor; GDNF, glial cell-derived neurotrophic factor; TGF, transforming growth factor.

compared with rNSCs, tsNSCs exhibited stronger GFAP positive immunoreactivity, however, the proportion of GFAP positive tsNSCs was not significantly different compared with rNSCs ( $\mathrm{P}=0.665$; Fig. 4A and B). NeuN immunofluorescence staining demonstrated that tsNSCs exhibited stronger NeuN positive reactivity compared with rNSCs (Fig. 4C). In addition, quantitative analysis demonstrated that the proportion of NeuN tsNSCs was markedly higher compared with rNSCs $(\mathrm{P}=0.0002$; Fig. 4D).

Positive expression of NT3, BDNF, GDNF and TGF $\beta 1$ in $t s N S C s$ and $r N S C s$. Immunofluorescence staining was also performed to detect the expression of NT3, BDNF, GDNF and TGF $\beta 1$ in rNSCs and tsNSCs. Compared with rNSCs, tsNSCs expressed stronger NT3 positive immunoreactivity, and the proportion of NT3 positive tsNSCs was markedly higher compared with rNSCs $(\mathrm{P}<0.01$; Fig. 5A and $\mathrm{B})$. In addition, the BDNF positive immunoreactivity in rNSCs was stronger compared with tsNSCs, and quantitative analysis showed that the proportion of BDNF positive tsNSCs was markedly lower compared with rNSCs ( $\mathrm{P}=0.045$; Fig. $5 \mathrm{~A}$ and $\mathrm{B})$. Compared with rNSCs, GDNF positive immunoreactivity in tsNSCs was higher. However, quantitative analysis of the proportion of GDNF positive cells indicated no significant difference between the two groups $(\mathrm{P}=0.173$; Fig. $5 \mathrm{~A}$ and $\mathrm{B})$. Furthermore, according to immunofluorescence staining analysis, the proportion of TGF $\beta 1$ tsNSCs was not significantly different compared with rNSCs $(\mathrm{P}=0.26$; Fig. $5 \mathrm{~A}$ and $\mathrm{B})$. The negative control exhibited no positive immunoreactivity (Fig. 5C).

\section{Discussion}

Two primary conclusions were drawn based on the results of the present study. The first is that the ability of tsNSCs to differentiate into neurons was stronger compared with rNSCs. In addition, the level of NT3 expression in tsNSCs was significantly higher compared with rNSCs, and the level of BDNF expression was lower in tsNSCs.

In the current study, as revealed by in vitro culture, the proliferation of tsNSCs was substantially slower compared 
with rNSCs. Concerning NSC culture, Pan et al (26) reported that NSCs from embryonic rats exhibited a higher number of NSCs and a markedly stronger proliferative ability compared with those from neonatal rats, therefore, they were more suitable for clinical therapy for nerve regeneration and recovery. Tian (27) demonstrated that goat embryonic stem cell (ESC)-like cells were similar to mice ESCs in colony morphology, however differences existed in the in vitro mode of passage. Additionally, these two cell types formed cell colonies only in the form of cell aggregates and the cloning efficiency may be substantially lower. In the present study, embryonic rats and tree shrews were employed as animal models to examine the proliferation ability of NSCs. A nestin antibody was used to demonstrate that the in vitro culture models of tsNSCs and rNSCs were successfully established. Comparison of the morphology of these two types of NSCs revealed that both formed clonal cell clumps that exhibited a suspended growth style with similar morphology, as they exhibited a round shape with strong refraction and clear boundaries. Both cell types grew into neurospheres, however, compared with rNSCs, tsNSCs grew and proliferated relatively slower. These results indicated that from rodents to primates, the proliferation ability of NSCs reduces.

Using immunofluorescence staining, the present study demonstrated that tsNSCs and rNSCs, induced by serum, differentiated into neurons and astrocytes. This indicates that both types of NSCs exhibit multi-differentiation properties. However, tsNSCs exhibited a stronger ability to differentiate into neurons compared with rNSCs. Previously, certain studies reported that low concentrations of serum promoted the differentiation of NSCs derived from neonatal rats into neurons, while high concentrations facilitated the differentiation of NSCs into neural glial cells, such as GFAP positive cells $(28,29)$. Additionally, the presence of neurotrophic factors in culture medium was reported to be important in the differentiation of bone marrow mesenchymal stem cells into neural stem cell-like cells (28-31). Furthermore, one study demonstrated that hypoxia promoted the differentiation of NSCs into neurons by activating the Notch signaling pathway (30). The results of the above studies indicate that rNSCs may be induced to differentiate into neurons and astrocytes under certain conditions. In the present study, the results demonstrated that the ability of tsNSCs to differentiate into glial cells was similar to rNSCs, however, tsNSCs exhibited a stronger ability to differentiate into neurons. To the best of our knowledge, there are few previous reports that have investigated the differences in the differentiation potentials between tsNSCs and rNSCs.

Further immunofluorescence experiments demonstrated that tsNSCs expressed a lower level of BDNF and a higher level of NT3 compared with rNSCs, while no significant differences were observed for GDNF and TGF $\beta 1$ expression between the two groups. BDNF is widely distributed in various brain areas, including the hippocampus, thalamus, amygdala and cortical layer, and has essential roles in the survival, growth and development of neurons $(32,33)$. Researchers demonstrated that the expression level of BDNF in adult tree shrews was substantially higher compared with the expression in fetal and neonatal tree shrews $(33,34)$. However, few studies have compared BDNF levels between rat and tree shrews. Based on the established functions of $\operatorname{BDNF}(32,33)$ and the stronger proliferation ability of rNSCs in the current study, we hypothesize that this increased proliferation ability may be associated with the higher expression of BDNF in rNSCs compared with tsNSCs. Notably, the NT3 expression level was higher in tsNSCs compared with rNSCs in the current study. It has been demonstrated that NT3 is associated with the differentiation of neurons in the CNS and peripheral nervous system, and the higher intelligence level of tree shrews compared with rats (34-36). The results of the present study indicate that the higher expression of NT3 in tsNSCs may be associated with the increased ability of tsNSCs to differentiate into neurons compared with rNSCs. Therefore, higher NT3 and lower BDNF expression in tsNSCs may contribute to the increased ability to differentiate into neurons and weaker proliferation ability in tsNSCs compared with rNSCs, which was observed in the present study.

Concerning the application of tree shrews in research, researchers have reported that tree shrews were useful and easier to work with compared with rats in a hepatitis B virus injection study (37). Furthermore, tree shrews were considered to be a good animal for depression studies, and they may be widely applied for the pathological and physiological investigation of depression $(38,39)$. Therefore, tree shrews may be a suitable alternative for rodents and may partially replace experiments on monkeys in the preparation of animal brain disease models.

In conclusion, the results of the current study demonstrated that, compared with rNSCs, tsNSCs exhibited a weaker proliferative ability, however, their ability to differentiate into neurons was much stronger. These results provide valuable evidence for the increased use of tree shrews as models for CNS diseases in humans.

\section{Acknowledgements}

The present study was supported by the Program of Innovative Research Team In Science and Technology in University of Yunnan and the Program of Innovative Research Team In Science and Technology in Yunnan Province and supported by a grant from the National Key Technology Research and Development Program of the Ministry of Science and Technology of China (grant no. 2014BAI01B10).

\section{Competing interests}

The authors declare that they have no competing interests.

\section{References}

1. Gage FH and Temple S: Neural stem cells: Generating and regenerating the brain. Neuron 80: 588-601, 2013.

2. Blurton JM, Kitazawa M, Martinez CH, Castello NA, Müller FJ, Loring JF, Yamasaki TR, Poon WW, Green KN and LaFerla FM: Neural stem cells improve cognition via BDNF in a transgenic model of Alzheimer disease. Proc Natl Acad Sci USA 106: 13594-13599, 2009.

3. Suksuphew S and Noisa P: Neural stem cells could serve as a therapeutic material for age-related neurodegenerative diseases. World J Stem Cells 7: 502-511, 2015.

4. Dooley D, Vidal P and Hendrix S: Immunopharmacological intervention for successful neural stem cell therapy: New perspectives in CNS neurogenesis and repair. Pharmacol Ther 141: 21-31, 2014.

5. Lu P, Jones LL, Snyder EY and Tuszynski MH: Neural stem cells constitutively secrete neurotrophic factors and promote extensive host axonal growth after spinal cord injury. Exp Neurol 181: $115-129,2003$. 
6. Bergström T and Forsbery NK: Neural stem cells: Brain building blocks and beyond. Ups J Med Sci 117: 132-142, 2012.

7. Stenudd M, Sabelström H and Frisén J: Role of endogenous neural stem cells in spinal cord injury and repair. JAMA Neurol 72: 235-237, 2015.

8. Engel U and Wolswijk G: Oligodendrocyte-type-2 astrocyte (O-2A) progenitor cells derived from adult rat spinal cord: In vitro characteristics and response to PDGF, bFGF and NT-3. Glia 16: 16-26, 1996.

9. Wang Y: Survial and migration of neural stem cells in rat spinal cord (unpublished PhD thesis). Ji Lin University, 2007.

10. Seaberg RM and van der Kooy D: Adult rodent neurogenic regions: The ventricular subependymacontains neural stem cells, but the dentate gyrus contains restricted progenitors J Neurosci 22: 1784-1793, 2002.

11. Hu Y: Proliferation and differentiation of neural stem cells from newborn mouse hippocampi in vitro. Chin J Tissue Eng Res 79-85, 2013.

12. Hu YR: In vitro culture, induction and differentiation of neura stem cells from rat embryo. J Clin Rehabil Tissue Eng Res: 3651-3655, 2009

13. Sun YX: Culture and differentiation of cynomolgus monkey neural stem cells. J Clin Rehabili Tissue Eng Res 8821-8824, 2009.

14. Zhao CL: Establishment of in vitro culture method of neural stem cells from human embryonic hippocampus. J Capital Univ Med Sci: 144-146, 2004.

15. Fan Y, Huang ZY, Cao CC, Chen CS, Chen YX, Fan DD, He J, Hou HL, Hu L, Hu XT, et al: Genome of the Chinese tree shrew. Nat Commun 4: 1426, 2013.

16. Fan Y, Yu D and Yao YG: Tree shrew database (TreeshrewDB) A genomic knowledge base for the Chinese tree shrew. Sci Rep 4 7145, 2014.

17. Reynolds BA and Weiss S: Generation of neurons and astrocytes from isolated cells of the adult mammalian central nervous system. Science 255: 1707-1278, 1992

18. Reynolds BA, Tetzlaff W and Weiss S: A multipotent EGF-responsive striatal embryonic progenitor cell produces neurons and astrocytes. J Neurosci 12: 4565-4574, 1992.

19. Fuchs E: Social stress in tree shrews as an animal model of depression: An example of a behavioral model of a CNS disorder. CNS Spectr 10: 182-190, 2005

20. Zhang D, Gao L, Zhang YX, Sun L, Feng Y, He YW, Xia XS and Zhang HT: Crucial factors for de novo establishment of long-term primary culture of tree shrew hepatocytes. Zoological Res 01: 24-30, 2009

21. Zhang JJ, Su JJ and Yang G: Two isolating and culturemethods of primary tupaia hepatocytes in vitro. Sichuan J Zoology 02: $168-171,2009$

22. Gong M, Li SQ and Li F: Primary culture and purification of cerebral astrocyte of tree shrew. Sheng Li Xue Bao 63: 89-92, 2011 (In Chinese)

23. Wu YZ: Culture in vitro and identification of neural stem cells from hippocampus of neonataltree shrew (unpublished $\mathrm{PhD}$ thesis). Guang Xi Medical University, 2013.

24. National Research Council (US) Institute for Laboratory Animal Research. Guide for the Care and Use of Laboratory Animals Washington (DC): National Academies Press (US); 1996.
25. Yang WQ: The sino-burmese tree colts guangxi said Burma in the monkey brain stereotaxic atlas. Guangxi Science and Technology Press, Nanning, pp47-69, 1990.

26. Pan LC, Yin ZS, Wang W, Hu Y, Gao RB, Wang ML and Ren YG: Comparative study on the characteristics of culture and differentiation of neural stem cells from fetal and neonatal rat. Chinese J Clin Rehabi 05: 25-27+195, 2006 (In Chinese).

27. Tian HB: Studies of establishment of mouse and goat embryonic stem cell lines and differentiation of them into neuron (unpublished PhD thesis). Shan Dong University, 2005.

28. Cao C: The in vitro culture of rat neural stem cells and its directed differentiation into neuron (D) (unpublished $\mathrm{PhD}$ thesis). Hei Bei Medical University, 2005

29. Xu P: Cultivation in vitro of rat neural stem cells and its conditioned medium effectson the differentiation of bone marrow derived mesenchymal stem cells into neural stem cells (unpublished PhD thesis). An Hui Medical University, 2014.

30. Wang SQ: Effects of Hypoxia on proliferation and differentiation of rats neural stem cells and analysis of signaling pathway (unpublished PhD thesis). Shang Hai University of Sport, 2013.

31. Wang LQ: Study on culture and differentiation of neural stem cells from neonatal rats in vitro (unpublished $\mathrm{PhD}$ thesis). $\mathrm{Su}$ Zhou University, 2004.

32. Gulino R, Lombardo SA, Casabona A, Leanza G and Perciavalle V: Levels of brain-derived neurotrophic factor and neurotrophin-4 in lumbar motoneurons after low-thoracic spinal cord hemisection. Brain Res 1013: 174-181, 2004.

33. He BL, Liu RW, Chen LL, et al: BDNF Expression in the Central Nervous System of Tree Shrews. J Kunming Med Univ 09: 21-23, 2011 (In Chinese)

34. Zhang D, Xiao Q, Luo $\mathrm{H}$ and Zhao KX: Effects of angiotensin-(1-7) on hippocampal expressions of GFAP and GDNF and cognitive function in rats with diabetes mellitus. Nan Fang Yi Ke Da Xue Xue Bao 35: 646-651, 2015 (In Chinese).

35. Jiao JL, Hao L, Zheng H, et al: The relation of BDNF Expression in the Central Nervous System and function of study of Tree Shrews. J Kunming Med Univ 05: 138, 2010 (In Chinese).

36. Jiao JL, He BL, Zheng H, LI B and Shen PQ: The comparative study of brain development among tree shrews (Tupaia blangeri chinensis), rhesus monkeys and rats. J Kunming Medical University 05: 39-41, 2010

37. Yan RQ, Su JJ, Huang DR, Gan YC, Yang C and Huang GH: Human hepatitis B virus and hepatocellular carcinoma. I. Experimental infection of tree shrews with hepatitis B virus. J Cancer Res Clin Oncol 122: 283-288, 1996.

38. Fuchs E, Czéh B and Flügge G: Examining novel concepts of the pathophysiology of depression in the chronic psychosocial stress paradigm in tree shrews. Behav Pharmacol 15: 315-325, 2004.

39. Wang J, Chai A, Zhou Q, Lv L, Wang L, Yang Y and Xu L: Chronic clomipramine treatment reverses core symptom of depression in subordinate tree shrews. PLoS One 8: e80980, 2013.

This work is licensed under a Creative Commons Attribution-NonCommercial-NoDerivatives 4.0 International (CC BY-NC-ND 4.0) License. 